\title{
Colloid-induced polymer compression
}

\author{
Alan R Denton ${ }^{1}$ and Matthias Schmidt ${ }^{2}$ \\ ${ }^{1}$ Department of Physics, North Dakota State University, Fargo, ND 58105-5566, USA \\ ${ }^{2}$ Institut für Theoretische Physik II, Heinrich-Heine-Universität Düsseldorf, Universitätsstraße 1, \\ D-40225 Düsseldorf, Germany \\ E-mail: alan.denton@ndsu.nodak.edu and mschmidt@thphy.uni-duesseldorf.de
}

Received 10 June 2002, in final form 9 August 2002

Published 8 November 2002

Online at stacks.iop.org/JPhysCM/14/12051

\begin{abstract}
We consider a model mixture of hard colloidal spheres and nonadsorbing polymer chains in a theta solvent. The polymer component is modelled as a polydisperse mixture of effective spheres, mutually noninteracting but excluded from the colloids, with radii that are free to adjust to allow for colloid-induced compression. We investigate the bulk fluid demixing behaviour of this model system using a geometry-based density functional theory that includes the polymer size polydispersity and configurational free energy, obtained from the exact radius-of-gyration distribution for an ideal (random-walk) chain. Free energies are computed by minimizing the free energy functional with respect to the polymer size distribution. With increasing colloid concentration and polymer-to-colloid size ratio, colloidal confinement is found to increasingly compress the polymers. Correspondingly, the demixing fluid binodal shifts, compared to the incompressible-polymer binodal, to higher polymer densities on the colloid-rich branch, stabilizing the mixed phase.
\end{abstract}

\section{Introduction}

The physical properties of soft-matter systems are often influenced by size polydispersity on mesoscopic length scales [1,2]. In colloidal suspensions, for example, variations in particle size can profoundly affect fluid-solid transitions [3]. In polymer solutions, chain length (degree of polymerization) often has a broad distribution. In practice, distributions in colloidal particle radius and polymer chain length often can be narrowed by physical selection or special synthesis methods. However, even monodisperse polymer chains have a radius of gyration that fluctuates in response to the surrounding environment. In this sense, polymers in solution are fundamentally polydisperse.

Polydispersity is especially relevant in mixtures of colloids and nonadsorbing (free) polymers, in which the polymer size sets the range of effective depletion-induced interactions between colloidal particles. A popular basis for modelling colloid-polymer (CP) mixtures is the Asakura-Oosawa (AO) model [4]. The AO model neglects fluctuations in chain 
conformations by approximating the polymers as effective spheres of fixed radius that are excluded from the hard-sphere (HS) colloids. The model further ignores polymer-polymer interactions by treating the polymer spheres as freely interpenetrating, an approximation best justified in a theta solvent.

Despite its simplicity, the AO model qualitatively captures thermodynamic phase behaviour observed in real $\mathrm{CP}$ mixtures - in particular, bulk fluid demixing into colloidrich and colloid-poor phases [5-8]. To address quantitative discrepancies with experiment, several recent studies have gone further to include polymer-polymer interactions-in both good solvents [9-11] and poor [12]—and polydispersity in polymer chain length [13-15]. In all of these studies, however, the polymers are modelled as effective spheres of fixed size (or fixed size distribution).

An issue that has not yet been widely explored is the influence of colloidal confinement on polymer conformations and implications for phase behaviour of CP mixtures. Polymers near surfaces and polymer-mediated interactions between surfaces have been examined by a variety of theoretical approaches, including scaling theory [16-18], integral equation theory [19, 20], classical density functional (DF) theory [21], and field-theoretic methods [22]. A far more challenging problem is the influence on polymers of confinement to a fluctuating random porous medium created by colloidal particles in suspension.

Numerical studies of colloids mixed with segmented polymer chains give some insight into polymer conformations in CP mixtures. Meijer and Frenkel [23] performed Monte Carlo simulations of hard spheres mixed with ideal (noninteracting) lattice-polymers. Dickman and Yethiraj [24] simulated mixtures of hard spheres and off-lattice polymer chains, modelled as freely jointed 'pearl necklaces' of hard spheres. Simulations such as these have revealed the potential importance of fluctuations in polymer size and shape in CP mixtures.

Here we consider an intermediate model in which the polymers maintain spherical shape but can adjust their radius-of-gyration distribution to the bulk colloid concentration. The constraint of fixed polymer size is thus relaxed by endowing the effective polymer spheres with an internal degree of freedom that can respond to colloidal confinement. We study the phase behaviour of a model mixture of HS colloids and compressible polymers by means of a geometry-based DF theory. Confinement-induced adjustments in polymer size are permitted by including in the free energy functional the free energy of ideal polymers, derived from the exact radius-of-gyration distribution of a random-walk chain.

The theory, when applied to bulk fluid phases, predicts both the demixing binodal and the polymer size distribution as a function of colloid and polymer concentrations. For sufficiently large polymer-to-colloid size ratio, the model displays bulk fluid demixing into colloid-rich and colloid-poor phases, qualitatively similar to the behaviour of the AO model. Upon demixing, however, the relatively unconfined polymers in the colloid-poor (vapour) phase coexist with compressed polymers in the colloid-rich (liquid) phase. We find that the polymer radius of gyration can be significantly reduced, by up to $20 \%$ or more, in the colloidal liquid phase. Correspondingly, the fluid demixing binodal shifts relative to the incompressible-polymer binodal.

Next, in section 2, we explicitly define the model system. In section 3 , we construct an appropriate classical DF theory that incorporates the conformational free energy of the polymer chains. Results for demixing phase diagrams and polymer size distributions are presented in section 4. Finally, we conclude in section 5.

\section{Model}

The model we consider is a generalization of the AO model to the case of compressible polymers. Explicitly, we consider a mixture of hard colloidal spheres (species $C$ ), of 
monodisperse radius $R_{C}$, and nonadsorbing, linear polymer chains (species $P$ ), monodisperse in length, but polydisperse in radius of gyration, $R_{P}$, in a volume $V$ at a temperature $T$ near the theta temperature. The restriction to a theta solvent allows the polymer chains to be reasonably approximated as noninteracting random walks. The colloids interact via a HS pair potential: $v_{C C}(r)=\infty$, if $r<2 R_{C}$, zero otherwise, where $r$ is the centre-centre interparticle distance. When interacting with colloids, each polymer is assumed to behave as an effective hard sphere of radius equal to its radius of gyration: $v_{C P}(r)=\infty$, if $r<R_{C}+R_{P}$, zero otherwise. Finally, interactions between polymers vanish for all distances: $v_{P P}(r)=0$.

To fix the polymer chemical potential, it is convenient to imagine a reservoir of pure polymer solution with which the system can freely exchange polymer (but not colloid) through a semi-permeable membrane. The reservoir also serves as a colloid-free reference state in which the polymer assumes an ideal radius-of-gyration distribution. Bulk fluid states are specified by the mean colloid and polymer (reservoir) number densities, $\rho_{C}$ and $\rho_{P}$ $\left(\rho_{P}^{r}\right)$, respectively. As bulk thermodynamic parameters, we use the colloid packing fraction, $\eta_{C}=(4 \pi / 3) \rho_{C} R_{C}^{3}$, and effective polymer packing fractions, $\eta_{P}=(4 \pi / 3) \rho_{P}\left(R_{g}^{r}\right)^{3}$ and $\eta_{P}^{r}=(4 \pi / 3) \rho_{P}^{r}\left(R_{g}^{r}\right)^{3}$, where $R_{g}^{r}$ denotes the root mean square (rms) radius of gyration in the reservoir, a quantity that is directly accessible in experiments. The reservoir polymer-to-colloid size ratio, $R_{g}^{r} / R_{C}$, provides a useful control parameter for tuning interparticle interactions, and thus thermodynamics.

\section{Theory}

\subsection{Density functional theory}

To investigate thermodynamic properties of the model system, we focus on the Helmholtz free energy as a functional of the inhomogeneous density profiles: the colloid density, $\rho_{C}(r)$, and a continuum of polymer densities, $\left\{\rho_{P}\left(r ; R_{P}\right)\right\}$, indexed by $R_{P}$ and normalized to the mean polymer density via $V^{-1} \int \mathrm{d}^{3} r \int_{0}^{\infty} \mathrm{d} R_{P} \rho_{P}\left(r ; R_{P}\right)=\rho_{P}$. It is convenient to separate the total free energy functional into three terms

$$
\begin{gathered}
F\left[\rho_{C}(\boldsymbol{r}),\left\{\rho_{P}\left(\boldsymbol{r} ; R_{P}\right)\right\}\right]=F_{\mathrm{id}}\left[\rho_{C}(\boldsymbol{r}),\left\{\rho_{P}\left(\boldsymbol{r} ; R_{P}\right)\right\}\right]+F_{\mathrm{ex}}\left[\rho_{C}(\boldsymbol{r}),\left\{\rho_{P}\left(\boldsymbol{r} ; R_{P}\right)\right\}\right] \\
+\int \mathrm{d}^{3} r \int_{0}^{\infty} \mathrm{d} R_{P} \rho_{P}\left(\boldsymbol{r} ; R_{P}\right) f_{\text {chain }}\left(\boldsymbol{r} ; R_{P} ;\left[\rho_{C}(\boldsymbol{r})\right]\right),
\end{gathered}
$$

where $F_{\text {id }}$ is the ideal free energy, $F_{\text {ex }}$ is the excess free energy in the AO model generalized to polydisperse (but incompressible) polymer, and $f_{\text {chain }}\left(\boldsymbol{r} ; R_{P} ;\left[\rho_{C}(\boldsymbol{r})\right]\right)$ is the local free energy of a compressible polymer chain. The third term in equation (1) stems from the internal degrees of freedom of the chains and is formally equivalent to the contribution of an external potential acting on the polymers. The ideal free energy, associated with (centre-of-mass) translational and mixing entropy of the colloids and polymers, is given exactly by

$$
\begin{aligned}
\beta F_{\text {id }}=\int \mathrm{d}^{3} r & \rho_{C}(\boldsymbol{r})\left[\ln \left(\rho_{C}(\boldsymbol{r}) \Lambda_{C}^{3}\right)-1\right] \\
& +\int \mathrm{d}^{3} r \int_{0}^{\infty} \mathrm{d} R_{P} \rho_{P}\left(\boldsymbol{r} ; R_{P}\right)\left[\ln \left(\rho_{P}\left(\boldsymbol{r} ; R_{P}\right) \Lambda_{P}^{3}\right)-1\right],
\end{aligned}
$$

where $\beta \equiv 1 / k_{\mathrm{B}} T, k_{\mathrm{B}}$ is Boltzmann's constant, and $\Lambda_{C}$ and $\Lambda_{P}$ are the respective colloid and polymer thermal wavelengths.

The excess free energy, arising from interactions, must be approximated. For this purpose, we adopt a geometry-based DF approach, which is immediately applicable to multi-component systems. As a basis, we start from a recently proposed DF theory [27] for a binary CP mixture 
in the AO model, the homogeneous limit of which is equivalent to the free volume theory of Lekkerkerker et al [7]. Here we generalize this theory [27] to mixtures of monodisperse colloids and polydisperse polymers. Following previous work [25, 27-29], the excess free energy is expressed in the form

$$
\beta F_{\mathrm{ex}}=\int \mathrm{d}^{3} x \Phi\left(\left\{n_{v}^{C}(\boldsymbol{x})\right\},\left\{n_{v}^{P}(\boldsymbol{x})\right\}\right),
$$

where the excess free energy density, $\Phi$, is a function of a set of weighted densities for colloid and polymer species. The weighted densities are defined as convolutions, with respect to geometric weight functions of the actual density profiles, and, in the case of polymers, integration over radius of gyration:

$$
\begin{aligned}
& n_{v}^{C}(\boldsymbol{x})=\int \mathrm{d}^{3} r \rho_{C}(\boldsymbol{r}) w_{v}\left(\boldsymbol{x}-\boldsymbol{r} ; R_{C}\right), \\
& n_{v}^{P}(\boldsymbol{x})=\int \mathrm{d}^{3} r \int_{0}^{\infty} \mathrm{d} R_{P} \rho_{P}\left(\boldsymbol{r} ; R_{P}\right) w_{v}\left(\boldsymbol{x}-\boldsymbol{r} ; R_{P}\right) .
\end{aligned}
$$

We use standard fundamental-measure weight functions $[25,26], w_{v}(r ; R), v=0,1,2,3, \mathrm{v} 1$, $\mathrm{v} 2, \mathrm{~m} 2$, for spheres of radius $R$, given by

$$
\begin{array}{ll}
w_{2}(\boldsymbol{r} ; R)=\delta(R-r), & w_{3}(\boldsymbol{r} ; R)=\Theta(R-r), \\
\boldsymbol{w}_{\mathrm{v} 2}(\boldsymbol{r} ; R)=\delta(R-r) \frac{\boldsymbol{r}}{r}, & \hat{\boldsymbol{w}}_{\mathrm{m} 2}(\boldsymbol{r} ; R)=\delta(R-r)\left(\frac{\boldsymbol{r} \boldsymbol{r}}{r^{2}}-\frac{\hat{\mathbf{1}}}{3}\right),
\end{array}
$$

where $r=|\boldsymbol{r}|, \delta(r)$ is the Dirac distribution, $\Theta(r)$ is the step function, and $\hat{\mathbf{1}}$ is the identity matrix. Further, linearly dependent weight functions are $w_{1}(r ; R)=w_{2}(r ; R) /(4 \pi R)$, $w_{0}(\boldsymbol{r} ; R)=w_{1}(\boldsymbol{r} ; R) / R$, and $\boldsymbol{w}_{\mathrm{v} 1}(\boldsymbol{r} ; R)=\boldsymbol{w}_{\mathrm{v} 2}(\boldsymbol{r} ; R) /(4 \pi R)$. The weight functions have dimensions (length) ${ }^{\nu-3}$ and differ in tensorial rank: $w_{0}, w_{1}, w_{2}$, and $w_{3}$ are scalars; $\boldsymbol{w}_{\mathrm{v} 1}$ and $\boldsymbol{w}_{\mathrm{v} 2}$ are vectors; and $\hat{\boldsymbol{w}}_{\mathrm{m} 2}$ is a (traceless) matrix.

The excess free energy density in equation (3) separates naturally into three parts:

$$
\Phi=\Phi_{1}+\Phi_{2}+\Phi_{3}
$$

which are defined as

$$
\begin{aligned}
\Phi_{1} & =\sum_{i=C, P} n_{0}^{i} \varphi_{i}\left(n_{3}^{C}, n_{3}^{P}\right), \\
\Phi_{2}= & \sum_{i, j=C, P}\left(n_{1}^{i} n_{2}^{j}-\boldsymbol{n}_{\mathrm{v} 1}^{i} \cdot \boldsymbol{n}_{\mathrm{v} 2}^{j}\right) \varphi_{i j}\left(n_{3}^{C}, n_{3}^{P}\right) \\
\Phi_{3}= & \frac{1}{8 \pi} \sum_{i, j, k=C, P}\left(\frac{1}{3} n_{2}^{i} n_{2}^{j} n_{2}^{k}-n_{2}^{i} \boldsymbol{n}_{\mathrm{v} 2}^{j} \cdot \boldsymbol{n}_{\mathrm{v} 2}^{k}\right. \\
& \left.\quad+\frac{3}{2}\left[\boldsymbol{n}_{\mathrm{v} 2}^{i} \hat{\boldsymbol{n}}_{\mathrm{m} 2}^{j} \boldsymbol{n}_{\mathrm{v} 2}^{k}-\operatorname{tr}\left(\hat{\boldsymbol{n}}_{\mathrm{m} 2}^{i} \hat{\boldsymbol{n}}_{\mathrm{m} 2}^{j} \hat{\boldsymbol{n}}_{\mathrm{m} 2}^{k}\right)\right]\right) \varphi_{i j k}\left(n_{3}^{C}, n_{3}^{P}\right) .
\end{aligned}
$$

Here tr denotes the trace operation and

$$
\varphi_{i \ldots k}\left(\eta_{C}, \eta_{P}\right)=\frac{\partial^{m}}{\partial \eta_{i} \cdots \partial \eta_{k}} \beta F_{0 \mathrm{~d}}\left(\eta_{C}, \eta_{P}\right)
$$

are derivatives of the zero-dimensional (0d) excess free energy, with

$$
\beta F_{0 \mathrm{~d}}\left(\eta_{C}, \eta_{P}\right)=\left(1-\eta_{C}-\eta_{P}\right) \ln \left(1-\eta_{C}\right)+\eta_{C} .
$$

Equations (3)-(13) completely specify the excess free energy in equation (1). 


\subsection{Application to bulk fluids}

For bulk fluid states, the density profiles are spatially constant. In the homogeneous limit $\left(\rho_{C}(\boldsymbol{r}) \rightarrow \rho_{C}\right.$ and $\left.\rho_{P}\left(\boldsymbol{r} ; R_{P}\right) \rightarrow \rho_{P}\left(R_{P}\right)\right)$, equation (1) yields the bulk fluid free energy density:

$$
\begin{aligned}
\frac{\beta F}{V}=\rho_{C}\left[\ln \left(\rho_{C} \Lambda_{C}^{3}\right)-1\right] & +\int_{0}^{\infty} \mathrm{d} R_{P} \rho_{P}\left(R_{P}\right)\left[\ln \left(\rho_{P}\left(R_{P}\right) \Lambda_{P}^{3}\right)-1\right] \\
& +\phi_{\mathrm{hs}}\left(\eta_{C}\right)-\int_{0}^{\infty} \mathrm{d} R_{P} \rho_{P}\left(R_{P}\right)\left[\ln \alpha\left(R_{P} ; \eta_{C}\right)-f_{\text {chain }}\left(R_{P} ; \eta_{C}\right)\right],
\end{aligned}
$$

which is still a functional of the polymer density distribution, $\rho_{P}\left(R_{P}\right)$. Here $\phi_{\mathrm{hs}}\left(\eta_{C}\right)$ is the excess free energy density of the pure HS system, given by

$$
\phi_{\mathrm{hs}}\left(\eta_{C}\right)=\frac{3 \eta_{C}\left[3 \eta_{C}\left(2-\eta_{C}\right)-2\left(1-\eta_{C}\right)^{2} \ln \left(1-\eta_{C}\right)\right]}{8 \pi R_{C}^{3}\left(1-\eta_{C}\right)^{2}},
$$

the same result as in the scaled-particle and Percus-Yevick compressibility approximations [30], and $\alpha\left(R_{P} ; \eta_{C}\right)$ is the free volume fraction, i.e., the fraction of the total volume not excluded to the polymer by the HS colloids, given implicitly by

$$
\ln \alpha\left(R_{P} ; \eta_{C}\right)=\ln \left(1-\eta_{C}\right)-\sum_{m=1}^{3} C_{m} \gamma^{m},
$$

where $\gamma=\eta_{C} /\left(1-\eta_{C}\right)$ and the coefficients are polynomials in the polymer-to-colloid size ratio, $q=R_{P} / R_{C}: C_{1}=3 q+3 q^{2}+q^{3}, C_{2}=9 q^{2} / 2+3 q^{3}$, and $C_{3}=3 q^{3}$.

It is worth noting that the free volume fraction is related to the polymer one-particle direct correlation function,

$$
c_{P}^{(1)}\left(\boldsymbol{r} ; R_{P} ;\left[\rho_{C}(\boldsymbol{r})\right]\right)=-\beta \frac{\delta F_{\mathrm{ex}}}{\delta \rho_{P}\left(\boldsymbol{r} ; R_{P}\right)},
$$

which has its physical origin in $\mathrm{CP}$ correlations. Substituting our DF approximation for $F_{\mathrm{ex}}$ into equation (17) and taking the homogeneous limit, we obtain

$$
c_{P}^{(1)}\left(R_{P} ; \eta_{C}\right)=-\sum_{v} \frac{\partial \Phi}{\partial n_{v}^{P}} * w_{v}\left(R_{P}\right)=\ln \alpha\left(R_{P} ; \eta_{C}\right),
$$

where $*$ denotes a convolution. Because our approximate excess free energy functional is linear in the polymer density, $c_{P}^{(1)}\left(R_{P} ; \eta_{C}\right)$ is independent of $\rho_{P}\left(R_{P}\right)$, depending only on the colloid density.

In equilibrium, the polymer density distribution in equation (14) is fixed by the EulerLagrange equation for the polymers:

$$
\frac{\delta(F / V)}{\delta \rho_{P}\left(R_{P}\right)}=\mu_{P}\left(R_{P}\right)
$$

where $\mu_{P}\left(R_{P}\right)$ is the chemical potential of polymers with radius of gyration $R_{P}$. Substituting equation (14) into (19), we have

$$
\ln \left(\rho_{P}\left(R_{P} ; \eta_{C}\right) \Lambda_{P}^{3}\right)-\ln \alpha\left(R_{P} ; \eta_{C}\right)+\beta f_{\text {chain }}\left(R_{P}\right)=\beta \mu_{P}\left(R_{P}\right),
$$

where $\rho_{P}\left(R_{P} ; \eta_{C}\right)$ is the equilibrium polymer density distribution at bulk colloid volume fraction $\eta_{C}$. Here we make the simplifying assumption that the chain free energy of a polymer with given $R_{P}$ in the system is the same as that of an equal-sized polymer in the reservoir, and thus independent of colloid concentration. Because the system and reservoir must be in 
equilibrium with respect to polymer exchange, the right side of equation (20) can be equated with the chemical potential of the corresponding polymer species in the reservoir:

$$
\mu_{P}\left(R_{P}\right)=\mu_{P}^{r}\left(R_{P}\right)=\beta^{-1} \ln \left(\rho_{P}^{r}\left(R_{P}\right) \Lambda_{P}^{3}\right)+f_{\text {chain }}\left(R_{P}\right),
$$

where $\rho_{P}^{r}\left(R_{P}\right)$ denotes the polymer density distribution in the reservoir. Further progress is facilitated by factoring the reservoir polymer density distribution, according to

$$
\rho_{P}^{r}\left(R_{P}\right)=\rho_{P}^{r} P^{r}\left(R_{P}\right)
$$

where $\rho_{P}^{r}$ and $P^{r}\left(R_{P}\right)$ are the mean density and radius-of-gyration probability distribution, respectively, of polymers in the reservoir. The advantage of equation (22) is that the probability distribution is simply related to the chain free energy via [31, 32]

$$
\beta f_{\text {chain }}\left(R_{P}\right)=-\ln P^{r}\left(R_{P}\right)+\text { constant independent of } R_{P},
$$

the arbitrary additive constant being chosen to normalize the distribution: $\int_{0}^{\infty} \mathrm{d} R_{P} P^{r}\left(R_{P}\right)=1$. Combining equations (20) and (21), and using (22) and (23), we obtain

$$
\rho_{P}\left(R_{P} ; \eta_{C}\right)=\rho_{P}^{r} \alpha\left(R_{P} ; \eta_{C}\right) P^{r}\left(R_{P}\right)=\alpha\left(R_{P} ; \eta_{C}\right) \rho_{P}^{r}\left(R_{P}\right) .
$$

The mean polymer densities in the system and reservoir are now seen to be related via

$$
\rho_{P}\left(\eta_{C}\right)=\int_{0}^{\infty} \mathrm{d} R_{P} \rho_{P}\left(R_{P} ; \eta_{C}\right)=\rho_{P}^{r} \alpha_{\mathrm{eff}}\left(\eta_{C}\right),
$$

where

$$
\alpha_{\mathrm{eff}}\left(\eta_{C}\right)=\int_{0}^{\infty} \mathrm{d} R_{P} \alpha\left(R_{P} ; \eta_{C}\right) P^{r}\left(R_{P}\right)
$$

is an effective free volume fraction. Equation (25) is a generalization of the incompressiblepolymer relation, $\rho_{P}\left(\eta_{C}\right)=\rho_{P}^{r} \alpha\left(\eta_{C}\right)$, from free volume theory [7,27]. Factoring $\rho_{P}\left(R_{P} ; \eta_{C}\right)$ according to

$$
\rho_{P}\left(R_{P} ; \eta_{C}\right)=\rho_{P}\left(\eta_{C}\right) P\left(R_{P} ; \eta_{C}\right),
$$

serves to define

$$
P\left(R_{P} ; \eta_{C}\right)=\frac{\alpha\left(R_{P} ; \eta_{C}\right)}{\alpha_{\text {eff }}\left(\eta_{C}\right)} P^{r}\left(R_{P}\right)
$$

as the normalized radius-of-gyration probability distribution of polymers in the system. Equation (28) makes manifest that the radius-of-gyration distribution in the system depends on colloid concentration and differs from the distribution in the reservoir.

Finally, substituting equations (23) and (24) into (14) and rearranging, we obtain the equilibrium bulk fluid free energy density,

$$
\frac{\beta F}{V}=\rho_{C}\left[\ln \left(\rho_{C} \Lambda_{C}^{3}\right)-1\right]+\rho_{P}^{r} \alpha_{\mathrm{eff}}\left(\eta_{C}\right)\left[\ln \left(\rho_{P}^{r} \Lambda_{P}^{3}\right)-1\right]+\phi_{\mathrm{hs}}\left(\eta_{C}\right) .
$$

Equations (28) and (29) are the main theoretical results of the paper and our basis for calculating polymer size distributions and phase behaviour in section 4. Practical implementation, however, first requires specification of $P^{r}\left(R_{P}\right)$.

\subsection{Radius-of-gyration probability distribution}

To approximate the radius-of-gyration probability distribution, we appeal to the statistical mechanics of a freely jointed chain. The configuration of a chain of $N$ links, each link of 
length $a$, can be specified by a set of position vectors, $\left\{\boldsymbol{R}_{i}\right\}=\left(\boldsymbol{R}_{0} \ldots \boldsymbol{R}_{N}\right)$, of the joints. For a given configuration, the radius of gyration, $R_{P}$, is defined by [31]

$$
R_{P}^{2}=\sum_{i=1}^{N}\left(\boldsymbol{R}_{i}-\boldsymbol{R}_{\mathrm{cm}}\right)^{2}
$$

where $\boldsymbol{R}_{\mathrm{cm}}=N^{-1} \sum_{i=1}^{N} \boldsymbol{R}_{i}$ is the centre-of-mass position of the chain. Averaged over configurations, the radius of gyration follows the probability distribution, $P^{r}\left(R_{P}\right)$. Moments of the distribution are defined as

$$
\left\langle R_{P}^{n}\right\rangle=\int_{0}^{\infty} \mathrm{d} R_{P} R_{P}^{n} P^{r}\left(R_{P}\right),
$$

the second ( $n=2$ ) moment being related to the rms radius of gyration of polymers in the reservoir [31, 32], $R_{\mathrm{g}}^{r}=\sqrt{\left\langle R_{P}^{2}\right\rangle}=a \sqrt{N / 6}$. For polymers in the system, the rms radius of gyration is defined as

$R_{\mathrm{g}}\left(\eta_{C}\right)=\left(\int_{0}^{\infty} \mathrm{d} R_{P} R_{P}^{2} P\left(R_{P} ; \eta_{C}\right)\right)^{1 / 2}=\left(\frac{1}{\alpha_{\mathrm{eff}}\left(\eta_{C}\right)} \int_{0}^{\infty} \mathrm{d} R_{P} R_{P}^{2} \alpha\left(R_{P} ; \eta_{C}\right) P^{r}\left(R_{P}\right)\right)^{1 / 2}$,

which clearly varies with bulk colloid concentration. Note that $R_{\mathrm{g}}\left(\eta_{C}=0\right)=R_{\mathrm{g}}^{r}$.

In general, the radius of gyration is a more appropriate measure of polymer size than the end-to-end displacement, which is not well defined for branched polymers. However, in contrast to the simple Gaussian distribution of the end-to-end displacement, the radiusof-gyration distribution is nontrivial, even for ideal chains. Flory and Fisk [33, 34] first proposed an empirical approximation for $P^{r}\left(R_{P}\right)$ based on the exact even moments calculated by Fixman [35]. Subsequently, Fujita and Norisuye [32,36] calculated the distribution exactly, obtaining the analytical result

$$
\begin{aligned}
P^{r}\left(R_{P}\right)=\frac{1}{\sqrt{2} \pi R_{\mathrm{g}}^{r} t^{3}} \sum_{k=0}^{\infty} \frac{(2 k+1) !}{\left(2^{k} k !\right)^{2}}(4 k+3)^{7 / 2} \exp \left(-t_{k}\right) \\
\quad \times\left[\left(1-\frac{5}{8 t_{k}}\right) K_{1 / 4}\left(t_{k}\right)+\left(1-\frac{3}{8 t_{k}}\right) K_{3 / 4}\left(t_{k}\right)\right],
\end{aligned}
$$

where $t=\left(R_{P} / R_{\mathrm{g}}^{r}\right)^{2}, K_{n}$ are the modified Bessel functions of the second kind, and $t_{k}=(4 k+3)^{2} /(8 t)$. To confirm equation (33), we have carried out Monte Carlo simulations of an ideal chain for $N=100$ and 1000 . Histograms of $R_{P}$, generated from $10^{6}$ independent configurations, are in essentially perfect agreement with the analytical expression [37].

Equation (33) specifies the radius-of-gyration probability distribution and thus, together with equation (23), the chain free energy. We emphasize that in modelling the chains as freely jointed, and so applying the ideal-chain distribution (equation (33)) to polymers mixed with colloids, we implicitly neglect any effect of colloidal confinement on the basic shape of the distribution. In particular, confinement-induced nonspherical distributions are neglected, although variation in the polymer size is allowed.

\section{Results: demixing phase behaviour and polymer size distribution}

The general conditions for phase coexistence are equality of the total pressure,

$$
p_{\text {tot }}=-\frac{F}{V}+\sum_{i=C, P} \rho_{i} \frac{\partial(F / V)}{\partial \rho_{i}}
$$



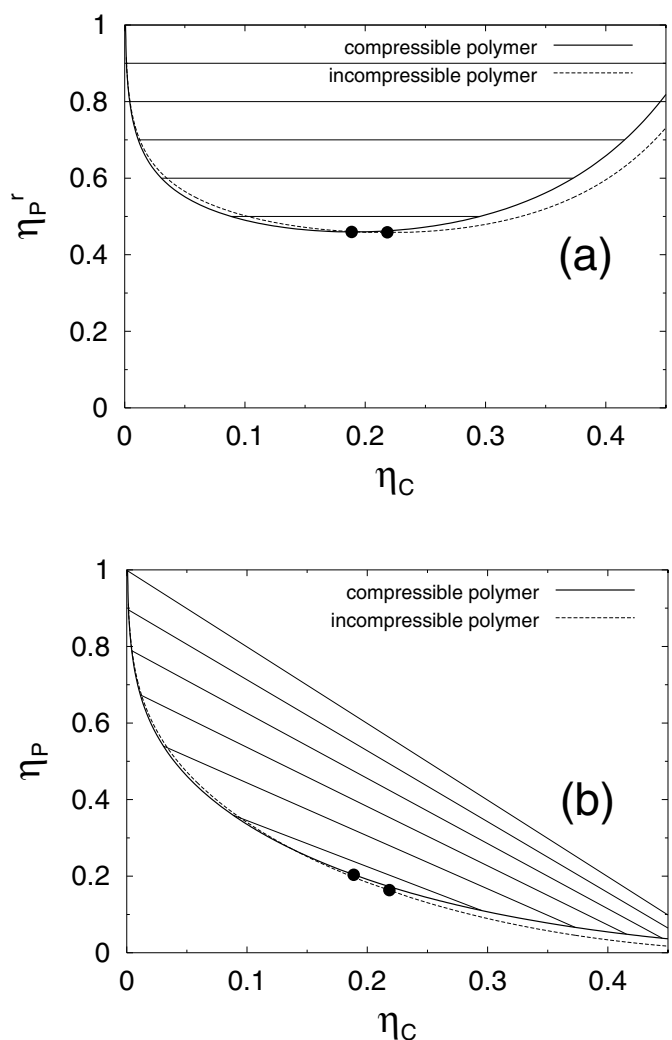

Figure 1. A fluid-fluid demixing phase diagram for $\mathrm{rms}$ reservoir polymer-to-colloid size ratio $R_{g}^{r} / R_{C}=0.5$. Solid curve: the binodal from the present theory, taking into account polymer compressibility; dashed curve: the binodal from free volume theory for the AO model with incompressible polymer. Thin straight lines indicate tie lines between coexisting phases; dots indicate critical points. (a) The reservoir representation: polymer reservoir packing fraction $\eta_{p}^{r}$ versus colloid packing fraction $\eta_{C}$; (b) the system representation: system polymer packing fraction $\eta_{P}$ versus $\eta_{C}$.

and of the chemical potentials for each species,

$$
\mu_{i}=\frac{\partial(F / V)}{\partial \rho_{i}}, \quad i=C, P,
$$

between the coexisting phases. Equilibrium between phases I and II requires $p_{\text {tot }}^{\mathrm{I}}=p_{\mathrm{tot}}^{\mathrm{II}}$ and $\mu_{C}^{\mathrm{I}}=\mu_{C}^{\mathrm{II}}$, equality of the polymer chemical potentials being enforced by equation (19).

In practice, bulk fluid phase diagrams can be computed as follows. The effective free volume fraction, $\alpha_{\text {eff }}\left(\eta_{C}\right)$, is first determined by substituting equations (16) and (33) into (26). Then, for a given reservoir polymer density, $\rho_{P}^{r}$, equations (29), (34), and (35) $(i=C)$ are solved numerically for the coexisting colloid packing fractions, $\eta_{C}^{\mathrm{I}}$ and $\eta_{C}^{\mathrm{II}}$. Finally, equation (25) converts from reservoir to system representation, giving the corresponding system polymer packing fractions, $\eta_{P}^{\mathrm{I}}$ and $\eta_{P}^{\mathrm{II}}$.

Figures 1 and 2 present bulk fluid phase diagrams for rms reservoir polymer-to-colloid size ratios $R_{g}^{r} / R_{C}=0.5$ and 1 , in both the system and reservoir representations. For comparison, we include demixing binodals both from the present theory, which takes into account polymer compressibility, and from the free volume theory of the AO model with incompressible polymer. For sufficiently high colloid and polymer packing fractions, the system demixes into colloid- 

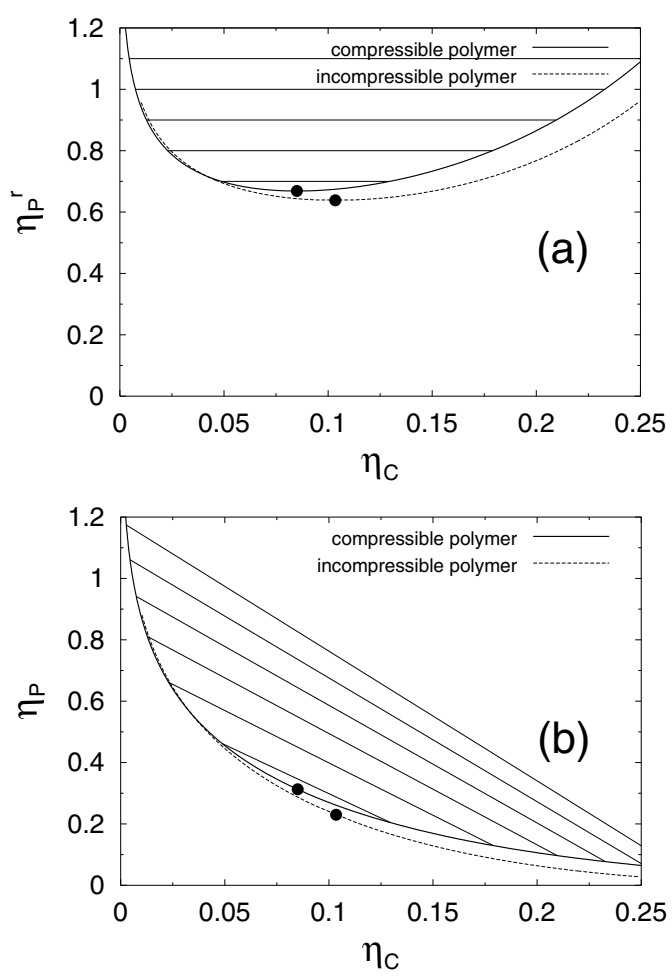

Figure 2. As figure 1, but for $R_{g}^{r} / R_{C}=1$. Note the change in scale compared with figure 1 .

rich (liquid) and colloid-poor (vapour) phases. We disregard here the liquid-solid branch of the phase diagram, since our size ratios are sufficiently high that fluid-fluid demixing can be assumed stable. The effect of polymer compressibility evidently is to shift the colloidal liquid branch of the binodal toward higher polymer density, stabilizing the system against demixing. Interpreted in terms of effective depletion-induced attraction between pairs of colloids, polymer compression shortens the range of attraction, tending to favour mixing. In passing, it may be anticipated that additional degrees of freedom allowing for nonspherical polymer conformations would tend to further stabilize the mixture if, as an alternative to bulk demixing, the polymer may simply distort its shape.

Figures 3 and 4 show the corresponding normalized polymer size distributions as a function of radius of gyration, scaled to the rms reservoir value. For given rms reservoir size ratio, with increasing colloid concentration, the distributions narrow and shift toward smaller radii, reflecting compression of the polymer by the confining colloids. In the case of $R_{g}^{r} / R_{C}=0.5$, the polymer is compressed, relative to its rms size in the reservoir, to $R_{g} / R_{g}^{r}=0.974,0.938$, 0.891 , and 0.829 at colloid packing fractions $\eta_{C}=0.1,0.2,0.3$, and 0.4 , respectively. For $R_{g}^{r} / R_{C}=1$, the polymer is even more strongly compressed to $R_{g} / R_{g}^{r}=0.915,0.826,0.738$, and 0.653 at the same respective values of $\eta_{C}$. Note that, for given $\eta_{C}, P\left(R_{P}\right)$ does not depend on $\eta_{P}$ (nor on $\eta_{P}^{r}$ ). Hence in the phase diagram, the polymer size distribution is constant along vertical lines (in both system and reservoir representations). What changes, of course, is the overall prefactor in $\rho_{P}\left(R_{P}\right)$. This invariance is an approximate feature of the theory resulting from the linearity of the excess free energy in polymer density. In reality, the presence of the polymers may alter the confining structure of the colloids, in turn modifying $P\left(R_{P}\right)$. 


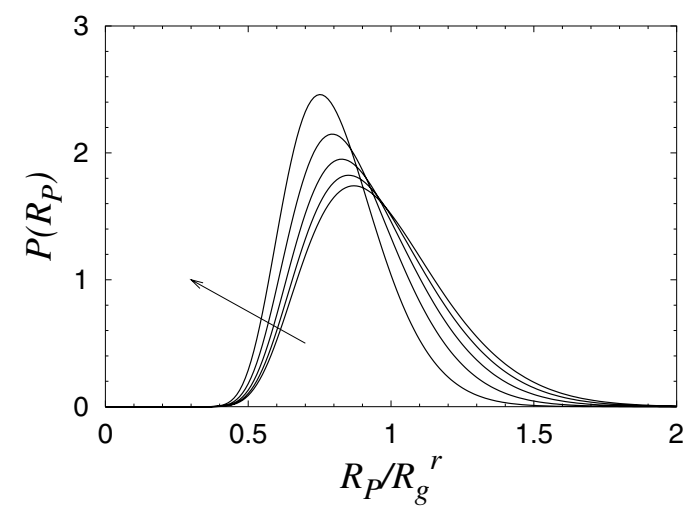

Figure 3. The normalized probability distribution, $P\left(R_{P}\right)$, of the scaled polymer radius of gyration, $R_{P} / R_{g}^{r}$ for rms reservoir polymer-to-colloid size ratio $R_{g}^{r} / R_{C}=0.5$ (corresponding to figure 1). The colloid packing fraction, $\eta_{C}$, increases in the direction of the arrow: $\eta_{C}=0,0.1,0.2,0.3$, and 0.4. Corresponding rms polymer size ratios decrease: $R_{g} / R_{g}^{r}=1,0.974,0.938,0.891$, and 0.829 .

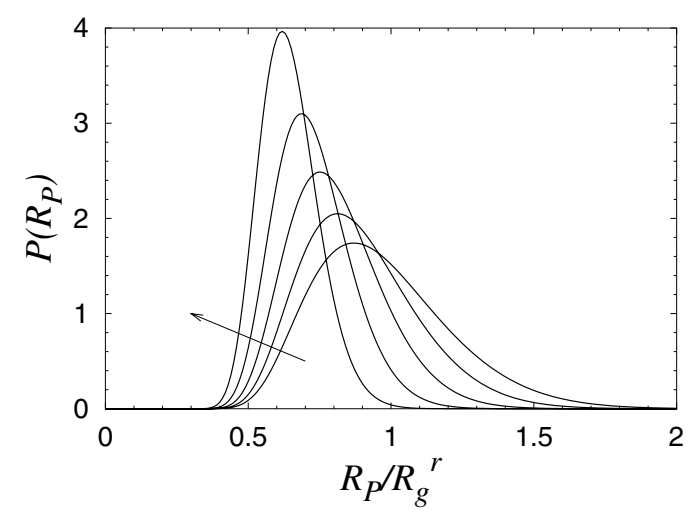

Figure 4. As figure 3, but for $R_{g}^{r} / R_{C}=1$ (corresponding to figure 2). In the direction of the arrow, the rms polymer size ratios are $R_{g} / R_{g}^{r}=1,0.915,0.826,0.738$, and 0.653 . Note the change in scale compared with figure 3.

\section{Conclusions}

We have investigated the bulk fluid demixing behaviour of a model mixture of hard colloidal spheres and nonadsorbing polymer chains in a theta solvent. The polymer component is modelled, on a mesoscopic level, as a polydisperse mixture of effective spheres with radii free to adjust to allow for colloid-induced compression. The model goes beyond previous studies by treating the polymer radius of gyration as an internal degree of freedom that is polydisperse and varies between coexisting phases. Like all effective-sphere models, however, our approach ignores details of the polymers on the segment level and thereby neglects any effects of polymer shape anisotropy.

To describe the model system, we have developed a geometry-based DF theory that incorporates polymer configurational free energy from consideration of the statistical mechanics of an ideal (random-walk) chain. For simplicity, the polymer chain free energy is assumed to be insensitive to colloidal confinement. Minimization of an approximate free 
energy functional with respect to the polymer size distribution yields both the equilibrium size distribution - modified by CP interactions - and the free energy, from which we compute bulk fluid phase diagrams. Polymer compression is found to increase with increasing colloid concentration and polymer-to-colloid size ratio. Correspondingly, the demixing fluid binodal shifts to higher polymer densities on the colloid-rich branch, favouring mixing.

Predictions of the theory may be tested by experiment and simulation. Although the predicted shift of the demixing binodal due to polymer compression would appear to improve agreement with limited experimental data near the theta point [8], more detailed measurements are certainly desirable. Polymer size distributions could be probed using either light scattering, by index matching the colloids (but not polymers) to the solvent, or neutron scattering by selectively deuterating the polymer (hydrocarbon) backbones. Detailed molecular simulations of segmented polymer chains mixed with hard spheres, along the lines of [23] and [24], could provide the clearest tests of our approximations and predictions.

Future applications to inhomogeneous CP mixtures under the influence of external potentials (due to walls, gravity, etc) and to liquid-solid transitions would be feasible and worthwhile. Extensions of the theory could explore the sensitivity of the polymer chain free energy to colloidal confinement, polydispersity in chain length, influences of anisotropic polymer conformations, distinctions between linear and branched polymers (as in colloid-starpolymer mixtures [38-40]), and effects of polymer nonideality in good and poor solvents.

\section{Acknowledgment}

A helpful discussion with Dr Michael Rubinstein is gratefully acknowledged.

\section{References}

[1] Daoud M and Williams C E (ed) 1999 Soft Matter Physics (Berlin: Springer)

[2] Sollich P 2002 J. Phys.: Condens. Matter 14 R79

[3] Bolhuis P G and Kofke D A 1996 Phys. Rev. E 54634

Kofke D A and Bolhuis P G 1999 Phys. Rev. E 59618

[4] Asakura S and Oosawa F 1954 J. Chem. Phys. 221255

[5] Gast A P, Russel W B and Hall C K 1986 J. Colloid Interface Sci. 109161

[6] Pusey P N 1991 Liquids, Freezing and Glass Transition ed J-P Hansen, D Levesque and J Zinn-Justin (Amsterdam: North-Holland)

[7] Lekkerkerker H N W, Poon W C K, Pusey P N, Stroobants A and Warren P B 1992 Europhys. Lett. 20559

[8] Ilett S M, Orrock A, Poon W C K and Pusey P N 1995 Phys. Rev. E 511344

[9] Warren P B, Ilett S M and Poon W C K 1995 Phys. Rev. E 525205

[10] Schmidt M, Denton A R and Brader J M 2002 Preprint

[11] Bolhuis P G, Louis A A and Hansen J-P 2002 Preprint

[12] Schmidt M and Denton A R 2002 Phys. Rev. E 65061410

[13] Sear R P and Frenkel D 1997 Phys. Rev. E 551677

[14] Warren P B 1997 Langmuir 134588

[15] Lee J-T and Robert M 1999 Phys. Rev. E 607198

[16] de Gennes P-G 1979 Scaling Concepts in Polymer Physics (Ithaca, NY: Cornell University Press)

[17] Joanny J F, Leibler L and de Gennes P-G 1979 J. Polym. Sci. 171073

[18] de Gennes P-G 1981 Macromolecules 141637

[19] Yethiraj A, Hall C K and Dickman R 1992 J. Colloid Interface Sci. 151102

[20] Chatterjee A P and Schweizer K S 1998 J. Chem. Phys. 10910464 Chatterjee A P and Schweizer K S 1998 J. Chem. Phys. 10910476

[21] Bechinger C, Rudhardt D, Leiderer P, Roth R and Dietrich S 1999 Phys. Rev. Lett. 833960

[22] Eisenriegler E, Hanke A and Dietrich S 1996 Phys. Rev. E 541134 Eisenriegler E 1997 Phys. Rev. E 553116

Hanke A, Eisenriegler E and Dietrich S 1999 Phys. Rev. E 596853 
[23] Meijer E J and Frenkel D 1991 Phys. Rev. Lett. 671110

Meijer E J and Frenkel D 1994 J. Chem. Phys. 1006873

Meijer E J and Frenkel D 1995 Physica A 213130

[24] Dickman R and Yethiraj A 1994 J. Chem. Phys. 1004683

[25] Rosenfeld Y 1989 Phys. Rev. Lett. 63980

[26] Tarazona P 2000 Phys. Rev. Lett. 84694

Cuesta J A, Martinez-Raton Y and Tarazona P 2002 J. Phys.: Condens. Matter 1411965

[27] Schmidt M, Löwen H, Brader J M and Evans R 2000 Phys. Rev. Lett. 851934

[28] Rosenfeld Y, Schmidt M, Löwen L and Tarazona P 1996 J. Phys.: Condens. Matter 8 L577

[29] Rosenfeld Y, Schmidt M, Löwen L and Tarazona P 1997 Phys. Rev. E 554245

[30] Hansen J P and McDonald I R 1986 Theory of Simple Liquids 2nd edn (London: Academic)

[31] Doi M and Edwards S F 1986 The Theory of Polymer Dynamics (Oxford: Clarendon)

[32] Yamakawa H 1970 Modern Theory of Polymer Solutions (New York: Harper \& Row)

[33] Flory P J and Fisk S 1966 J. Chem. Phys. 442243

[34] Flory P J 1969 Statistical Mechanics of Chain Molecules (New York: Wiley)

[35] Fixman M 1962 J. Chem. Phys. 36306

[36] Fujita H and Norisuye T 1970 J. Chem. Phys. 521115

[37] Schmidt M and Denton A R 2002 unpublished

[38] Poon W C K, Egelhaaf S U, Stellbrink J, Allgaier J, Schofield A B and Pusey P N 2001 Phil. Trans. R. Soc. A 359897

[39] Dzubiella J, Jusufi A, Likos C N, von Ferber C, Löwen H, Stellbrink J, Allgaier J, Richter D, Schofield A B, Smith P A, Poon W C K and Pusey P N 2001 Phys. Rev. E 6410401

[40] Dzubiella J, Likos C N and Löwen H 2002 J. Chem. Phys. 1169518 